\title{
ESTUDO COMPARATIVO DA REAÇÃO INFLAMATÓRIA RENALENTRE ÁLCOOL DE POLIVINIL-FLOCULAR E ÁLCOOL DE POLIVINIL + ACETATO DE POLIVINIL - ESFÉRICO: ESTUDO EXPERIMENTAL
}

\author{
COMPARATIVE STUDY OF RENAL INFLAMMATORY REACTION BETWEEN \\ IRREGULAR - POLYVINYL ALCOHOL AND SPHERICAL - POLYVINYL ALCOHOL + \\ POLYVINYL ACETATE: EXPERIMENTAL STUDY
}

\author{
Warley Dias Siqueira Mendes, ACBC-RJ' ${ }^{1}$, Vera Lucia Antunes Chagas²; \\ José Carlos Pinto ${ }^{3}$; José Guilherme Caldas ${ }^{4}$; Gaudencio Espinosa,TCBC-RJ ${ }^{5}$
}

\begin{abstract}
RESUMO: Objetivo: Avaliar as características e os efeitos de um agente embólico, disponível comercialmente, consistindo de Polivinil Álcool (PVA) de morfologia flocular, e comparar com um agente esférico, de tecnologia nacional, consistindo de Polivinil Álcool e Polivinil Acetato (PVA + PVAc). Método: Foram utilizadas fêmeas de coelho albino "New Zealand", submetidas à embolização arterial renal. PVA-flocular foi usado em 24 animais, assim como PVA+PVAc-esférico. Seis animais foram utilizados como controle. Todos foram mantidos em cativeiro até a morte, por períodos pós-operatórios de 48 horas, cinco dias, 10 dias e 30 dias. Resultados: Ambos os agentes promoveram oclusão do vaso e infarto do órgão. O estudo microscópico inicial das artérias embolizadas com PVA-flocular, mostra oclusão com trombo e PVA. Os vasos embolizados com PVA+PVAc-esférico, mostram os agentes ocupando praticamente todo o lúmen. No estudo de 30 dias, observa-se absorção do trombo e retração dos agentes de PVAflocular, criando espaços. E com PVA+PVAc-esférico, pode-se observar os agentes circundados por intensa fibrose. Conclusões: Ambas as partículas foram efetivas para causar isquemia tecidual. A reação inflamatória foi mais intensa com PVA+PVAc-esférico que também apresentou grau de penetração maior no sistema vascular (Rev. Col. Bras. Cir. 2005; 32(3): 120-126).
\end{abstract}

Descritores: Experimentação animal; Embolização terapêutica; Álcool de polivinil; Radiologia intervencionista; Estudo comparativo.

\section{INTRODUÇÃO}

Nos últimos anos, procedimentos de embolização transcateter têm se destacado cada vez mais no tratamento de grande variedade de doenças ${ }^{1}$, com bons resultados. $\mathrm{O}$ sucesso obtido com a embolização no tratamento de várias condições, tais como, leiomioma uterino, hemoptise e malignidades hepáticas e a sua importância como coadjuvante pré-operatório no tratamento de meningiomas intracranianos ou malformações vasculares, tem despertado o interesse neste procedimento. Interesse este que tem encorajado recentes estudos sobre o mecanismo de ação dos diferentes tipos de agentes embólicos e estimulado o desenvolvimento de novos agentes, com o objetivo de se alcançar melhores resultados no tratamento com embolização.

Os materiais que atualmente existem ainda possuem algumas características indesejáveis (superfície irregular, má calibragem e custo elevado). Desta forma ainda existe a necessidade de desenvolver materiais mais adequados. A Coordenação dos Programas de Pós-Graduação de Engenharia da Universidade Federal do Rio de Janeiro (COPPE/UFRJ) desenvolveu uma partícula de melhores características (superfície esférica, bem calibrada e menor custo).
O objetivo deste estudo é comparar a resposta tecidual da embolização intra-arterial de rins de coelhos, avaliando comparativamente as partículas de Polivinil Álcool que existem comercialmente (PVA-flocular) e as partículas de Polivinil Álcool + Polivinil Acetato Esférico (PVA+PVAc-esférico), desenvolvidas pela COPPE/UFRJ.

\section{MÉTODO}

Este estudo foi aprovado pela Comissão de Ética em Pesquisa (C.E.P.A.) em Animais do Departamento de Cirurgia da UFRJ. Foram utilizadas fêmeas de coelho albino "New Zealand", pesando entre $2.300 \mathrm{gr}$ e $3.200 \mathrm{gr}$, que foram submetidas a embolização arterial renal, sob anestesia intramuscular, divididas em dois grupos de 24 animais. Um destes foi embolizado com PVA-flocular (Trufill ${ }^{\circledR}$, Cordis, Miami, EUA) e o outro com PVA+PVAc-esférico (COOPE/UFRJ), solicitação de patente número P10404994-2 (Figura 1). Os grupos foram subdivididos em quatro subgrupos de seis animais $(\mathrm{n}=6)$ e mantidos em cativeiro até a morte, por períodos pós-operatórios de 48 horas, cinco dias, 10 dias e 30 dias, respectivamente (Tabela 1).

Um grupo adicional de simulação ("sham"), para controle da técnica de cateterização renal, foi dividido em

1. Mestre em Medicina, área de concentração Cirurgia Geral, Setor Cirurgia Vascular, pela Faculdade de Medicina da UFRJ

2. Professora Assistente do Departamento de Patologia da UFRJ

3. Programa de Engenharia Química - COPPE/UFRJ

4. Departamento de Radiologia do INCOR/HC-USP

5. Professor Adjunto de Cirurgia da UFRJ

Recebido em 15/12/2004

Aceito para publicação em 03/03/2005

Estudo realizado nos Departamentos de Cirurgia, Radiologia e Anatomia Patológica da Universidade Federal do Rio de Janeiro. 

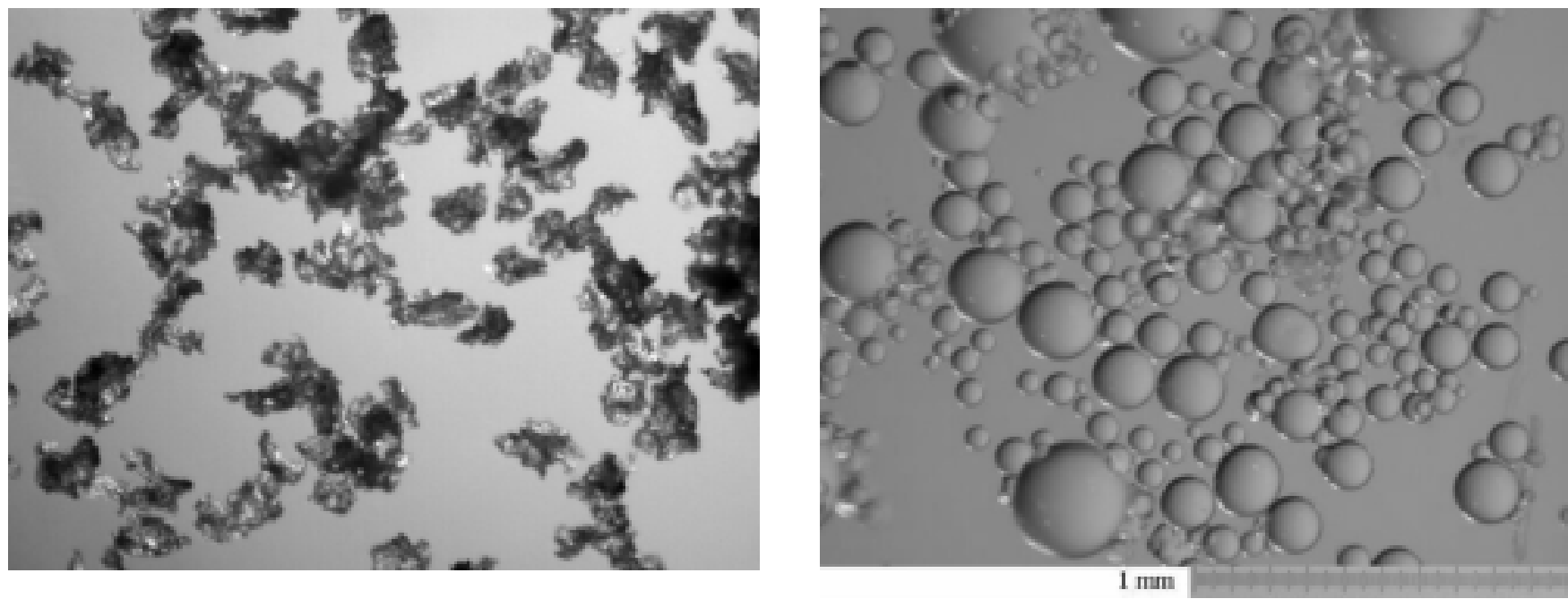

Figura 1 - Imagens de microscopia, onde observamos as partículas de PVA-flocular (A), que existe comercialmente (Trufill ${ }^{\circledR}$, Cordis, EUA) $e$ as partículas de PVA+PVAc-esférico (B), desenvolvidas pela COPPE/UFRJ.

quatro subgrupos de três animais $(n=3)$, e mantidos até a morte por igual período de tempo dos grupos submetidos a embolização (Tabela 1).

Os animais foram mantidos no biotério do laboratório de Cirurgia Experimental, do Departamento de Cirurgia da UFRJ, em gaiolas apropriadas para sua espécie. A dieta consistiu em ração industrial, do tipo Linha Natural Purina p/ Coelhos (Agribrands do Brasil Ltda.-SP)

Após a morte, foram retirados os rins submetidos à embolização, assim como os contralaterais, como controle, e enviados para análise anatomopatológica para posterior estudo comparativo.

Foi utilizado Cloridrato de Ketamina (Vetanarcol ${ }^{\circledR}$, König), na dose de 40mg/kg e Xilazina, na dose de $2 \mathrm{mg} / \mathrm{kg}$, via intramuscular. Também foi utilizado Cloridrato de Lidocaína a $1 \%$, no local da incisão. O animal foi colocado em decúbito dorsal, com os quatro membros contidos. Realizada tricotomia da região inguinal direita, com posterior antissepsia da pele, com solução iodada e colocação de campos operatórios esterilizados. Realizado acesso cirúrgico inguinal, com identificação e reparo da artéria femoral direita. Heparinização plena na dose $100 \mathrm{UI} / \mathrm{Kg}$, via intra-arterial.

O estudo fluoroscópico e angiográfico foi realizado com arco em “C”, unidade de fluoroscopia móvel, BV-300 (Philips, Holanda). Introduzido cateter angiográfico de polietileno $\left(\mathrm{DAV}^{\circledR}\right.$, Cook, Bloomington, IN, USA) de 4, 1 French, apoiado sobre fioguia hidrofílico (Roadrunner ${ }^{\circledR}$, Cook, Bloomington, IN, USA). Em seguida foi feito cateterismo seletivo da artéria renal direita, sob fluoroscopia, com injeção de contraste iodado (Reliev ${ }^{\circledR}$, Justesa) para obtenção de estudo angiográfico renal pré-procedimento avaliando as fases arterial, parenquimatosa e de retorno venoso. Posteriormente, foi realizada embolização intra-arterial com partículas de PVA-flocular ou PVA+PVAc-esférico, de 150 a 250 mm de diâmetro, suspensas em $20 \mathrm{ml}$ de soro fisiológico a $0,9 \%$, de acordo com o peso corporal dos animais (Tabela 2).

Posteriormente foi realizado estudo angiográfico de controle para avaliar o efeito do procedimento sobre a vascularização do rim. Depois de retirado o cateter angiográfico, foi realizada ligadura proximal e distal da artéria femoral. Revisão da hemostasia e fechamento da pele com sutura contínua, com fio monofilamentar 4-0 (Mononylon ${ }^{\circledR}$ ).

Os animais foram mortos através de injeção intravenosa de tiopental e cloreto de potássio, na veia marginal da orelha. Foram retirados ambos os rins (o embolizado e o contralateral), mediante laparotomia mediana. Depois de seccionados ao meio longitudinalmente (sentido coronal), foram imediatamente imersos e mantidos em solução de formaldeído a $10 \%$.

O estudo histopatológico foi feito com microscopia óptica convencional. Fragmentos com cerca de $3 \mathrm{~mm}$ obtidos do rim embolizado e do contralateral. Realizados cortes de $5 i ̀ m$ de espessura, que foram submetidos às técnicas de coloração, pelos métodos de hematoxilina-eosina (HE) e tricrômico de Masson. A observação foi realizada em microscópio óptico (Leitz-Dialux), no serviço de Anatomia Patológica do Hospital Universitário Clementino Fraga Filho da UFRJ.

\section{Tabela 1}

\begin{tabular}{|c|c|c|c|c|c|}
\hline \multicolumn{2}{|c|}{$\begin{array}{l}\text { Grupo de Simulação } \\
\text { (SHAM n=12) }\end{array}$} & \multicolumn{2}{|c|}{$\begin{array}{c}\text { Grupo PVA-flocular } \\
(n=24)\end{array}$} & \multicolumn{2}{|c|}{$\begin{array}{c}\text { Grupo PVA+PVAc-esférico } \\
(n=24)\end{array}$} \\
\hline Subgrupo & $(n)$ & Subgrupo & $(n)$ & Subgrupo & $(n)$ \\
\hline 48 horas: & $(n=03)$ & 48 horas: & $(n=06)$ & 48 horas: & $(\mathrm{n}=06)$ \\
\hline 5 dias: & $(n=03)$ & 5 dias: & $(n=06)$ & 5 dias: & $(n=06)$ \\
\hline 10 dias: & $(n=03)$ & 10 dias: & $(n=06)$ & 10 dias: & $(n=06)$ \\
\hline 30 dias: & $(n=03)$ & 30 dias: & $(n=06)$ & 30 dias: & $(n=06)$ \\
\hline
\end{tabular}




\section{RESULTADOS}

Como resultado da técnica cirúrgica e do procedimento de embolização, todos os rins foram cateterizados seletivamente sem maiores dificuldades. Quanto ao processo de embolização, não houve agregação nem aglutinação na passagem das partículas, tanto com as partículas de PVA-flocular quanto com as partículas de PVA+PVAc-esférico, através do cateter 4-F. No estudo angiográfico de controle final, observou-se oclusão da circulação renal em todos os animais.

Nos rins com 48 horas de embolização notava-se palidez da superfície cortical subcapsular e, ao corte, discreto apagamento dos limites córtico-medulares, associado a leve hiperemia da medular. Tais alterações acentuaram-se proporcionalmente nos rins com cinco e 10 dias de embolizados, com palidez também na medular, até a completa perda dos limites córtico-medulares, quando comparados aos rins contralaterais utilizados como controles. Nos rins embolizados com as partículas esféricas este aspecto mostrava-se menos homogêneo, com áreas acastanhadas principalmente peri-hilares.

Os rins com 30 dias de embolizados mostraram diminuição significativa do volume, mais acentuada nos animais submetidos à embolização com as partículas esféricas. Os rins embolizados com PVA-flocular tinham a superfície cortical externa e a superfície de corte com aspecto semelhante ao observado com 10 dias de embolização. Porém, nos embolizados com esferas, a cortical externa mostrava depressões cicatriciais e cápsula renal espessada e aderida; ao corte notavam-se áreas homogêneas de aspecto cartilaginoso e outras serpiginosas amareladas.

No estudo da microscopia de 48 horas, observamos a presença das partículas de PVA-flocular e PVA+PVAc-esférico no interior dos vasos entremeadas com hemácias e esparsos polimorfonucleares (PMN), ambos mais numerosos com o PVAflocular (Figura 2). Com este produto, notavam-se alterações isquêmicas da parede arterial com área focal de ruptura e com afluxo de PMN, não observadas nos vasos embolizados com PVA+PVAc-esférico.
Tabela 2

\begin{tabular}{cc}
\hline Peso Corporal & $\begin{array}{c}\text { Dose de PVA-flocular ou } \\
\text { PVA+PVAc-esférico }\end{array}$ \\
\hline $2.300 \mathrm{~g} \mathrm{a} 2.600 \mathrm{~g}$ & $15 \mathrm{mg}$ \\
$2.600 \mathrm{~g} \mathrm{a} 2.900 \mathrm{~g}$ & $17,5 \mathrm{mg}$ \\
$2.900 \mathrm{~g} \mathrm{a} 3.200 \mathrm{~g}$ & $20 \mathrm{mg}$ \\
\hline
\end{tabular}

Com ambas as partículas observou-se, ao nível do córtex e da medular, resposta isquêmica importante com picnose nuclear, cariorrexis e cariólise, além de vacuolização e fragmentação citoplasmática em células tubulares e glomerulares, associados à congestão (Tabela 3). Estas alterações foram visualizadas nos dois grupos de animais, sendo que nos embolizados com partículas esféricas, notaram-se áreas preservadas correspondentes às regiões acastanhadas descritas na macroscopia. Havia ainda infiltrado inflamatório em faixa, constituído por PMN íntegros ou degenerados, no córtex subcapsular.

No $5^{\circ}$ dia observamos, nos rins das partículas de PVA-flocular, numerosos PMN e hemácias entre as partículas, as quais se mostravam vacuolizadas (Tabela 3). A parede vascular mostrava áreas focais de permeação por PMN, relacionados à lise celular. Observava-se ainda na região cortical subcapsular infiltração por PMN, acentuação do processo de necrose celular e focos de calcificação.

Com as partículas de PVA+PVAc-esférico observamos acentuado infiltrado inflamatório de PMN subcapsular, assim como a permeação por estas células das demais áreas do órgão, onde as alterações necróticas mostravam-se mais acentuadas que no período anterior (Tabela 3). Associavamse múltiplos focos de calcificação principalmente na medular, onde observou-se ainda acentuada congestão e regeneração do epitélio tubular, em meio a tecido de granulação intersticial. As partículas de PVA+PVAc-esférico preenchiam praticamente todo o lúmen vascular, entremeadas com hemácias ou por trombos em organização.
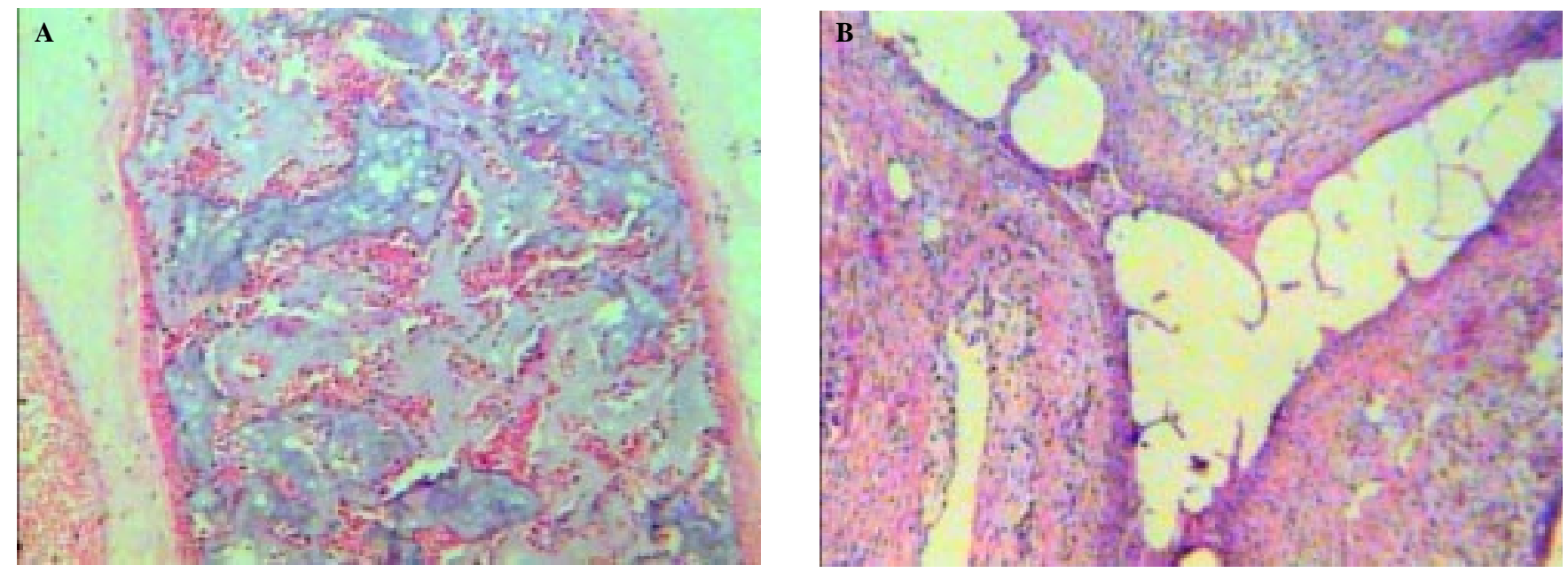

Figura 2 - Coloração pelo método de hematoxilina-eosina (HE) às 48 horas, onde observamos grande quantidade de hemácias (trombo) entre as partículas de PVA-flocular (A), enquanto que as partículas de PVA+PVAc-esférico preenchem praticamente todo o lúmen vascular (B). Notar a grande vacuolização das partículas de PVA-flocular, refletindo sua alta porosidade (A). 


\section{Tabela 3}

\begin{tabular}{cccc}
\hline Tempo & Fase & PVA-Comercial & PVA-Esférico \\
\hline 24-48h: & Isquemia Aguda & $++++/ 4+$ & $++++/ 4+$ \\
5 dias: & Inflamação Aguda (Neutrófilos) & Intra-Vascular & Tecidual e Cápsula \\
10 dias: & Inflamação Crônica (Linfócitos) & Atrofia da Parede Vascular & Hipertrofia da Parede Vascular \\
30 dias: & Fibrose & Moderada & Intensa \\
\hline
\end{tabular}

Após 10 dias, os vasos embolizados com partículas de PVA-flocular mostravam necrose da parede, por vezes relacionada à PMN; as partículas eram intensamente vacuolizadas e retraídas. Ao nível da cortical e medular, as alterações isquêmicas mostravam-se proeminentes, com PMN organizados em faixa subcapsular e ocasionais focos de mononucleares (linfócitos).

Com as partículas de PVA+PVAc-esférico observamos intenso infiltrado de PMN subcapsular em faixa e em torno das partículas, presença de trombos organizados. Ao nível da cortical e medular eram visíveis alterações isquêmicas importantes associadas à congestão, com múltiplos focos de calcificação, fibrose intersticial e regeneração do epitélio tubular especialmente na medular. Notavam-se algumas áreas preservadas entremeadas com focos de isquemia.

Nos rins com partículas de PVA-flocular, observamos aos 30 dias, retração importante das partículas de PVA (Figura 3A), permeadas por depósito de cálcio, com adelgaçamento e fibrose da parede vascular (Tabela 3). Na cortical persistiam as alterações isquêmicas associadas à deposição de cálcio. Na coloração pelo método de Masson, observou-se a presença de escassa fibrose subcapsular, cortical e medular, onde notavam-se túbulos com epitélio regenerado.

Nesta etapa, as partículas de PVA+PVAc-esférico mostravam-se circundadas por trombos organizados; na proximidade destes vasos notavam-se acúmulos focais de células inflamatórias mononucleares. Chamava a atenção, a calcificação presente nas regiões cortical e medular associada à presença de fibrose intensa (Figura 3B). Encontramos ainda extensas áreas de necrose isquêmica, epitélio de túbulos medulares regenerados e pequenas áreas ainda com parênquima preservado.

\section{DISCUSSÃO}

A técnica de embolização consiste na injeção de material sintético pós cateterismo seletivo dos vasos sangüíneos que irrigam a área comprometida de modo a ocluir mecanicamente a corrente sangüínea que nutre a região a ser tratada. $\mathrm{Na}$ atualidade, embolização intravascular constitui importante estratégia no combate a tumores, aneurismas e malformações vasculares $^{2}$. O rim foi escolhido por ser órgão com vascularização terminal e de apresentação bilateral, o que facilita o estudo comparativo entre o rim submetido à embolização e o rim contra-lateral de controle, não embolizado, e ainda permitir a manutenção das funções vitais do animal.

A taxa de sucesso que vem sendo apresentada recentemente fez surgir o interesse médico neste procedimento e nas substâncias utilizadas para tal, estimulando conseqüentemente o conhecimento do mecanismo de ação dos agentes embólicos disponíveis e o desenvolvimento de novos agentes para otimizar o sucesso terapêutico ${ }^{1}$. Diferentes materiais têm sido relatados na literatura mundial como úteis para embolização tais como, molas metálicas, borracha de silicone, microesferas de carbono e partículas de $\mathrm{PVA}^{3}$. Em todos os casos deve-se observar a compatibilidade entre a granulometria
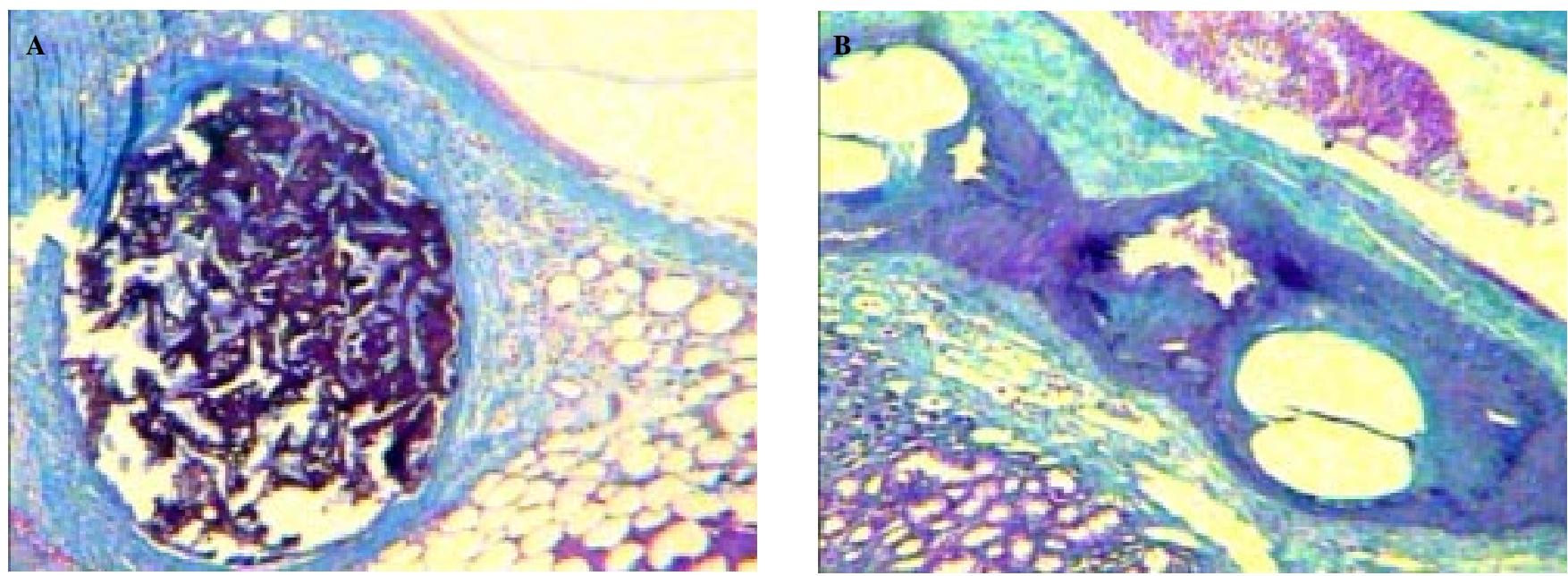

Figura 3 - Coloração pelo método de tricrômico de Masson aos 30 dias, onde nos rins com partículas de PVA-flocular observamos grande retração das partículas de PVA (A), com absorção do trombo vascular, criando espaços entre as partículas (A). Também notamos importante adelgaçamento da parede vascular (A). Com as partículas de PVA+PVAc-esférico observamos, nesta etapa, que as mesmas se apresentavam circundadas por fibrose intensa $(B)$, levando a um espessamento da parede vascular. 
do material a ser injetado com os diâmetros dos cateteres utilizados e dos vasos a serem tratados, a resposta local imediata e tardia, o tempo de permanência no local desejado. Dentre as diversas substâncias utilizadas, as partículas de PVA são os agentes embólicos mais utilizados, com biocompatibilidade, eficácia e relativa permanência bem documentadas ${ }^{4,5}$. As partículas de PVA são partículas inertes que apresentam boa compatibilidade, boa elasticidade e boa resistência química a ácidos, bases e detergentes ${ }^{5,6}$. Entretanto, as partículas de PVA existentes comercialmente apresentam alguns aspectos indesejáveis como superfície irregular, o que facilita a sua aglutinação e agregação, muitas vezes dificultando sua passagem pelo cateter angiográfico ou limitando o grau de sua progressão dentro da lesão vascular. Somado a isto, as partículas de PVA-flocular são suscetíveis à degradação ao longo do tempo (biodegradabilidade), facilitando a futura recanalização do leito vascular tratado ${ }^{1,4,7-9}$.

Com intuito de se contornar as características indesejáveis inerentes às partículas de PVA comercialmente disponíveis, formas diferentes de uso têm sido experimentadas e várias novas substâncias têm sido criadas e testadas ${ }^{1,4,8-15}$. Derdeyn et al. ${ }^{8}$ realizaram um estudo com objetivo de avaliar as características da partícula de PVA-flocular e determinar se ela muda de tamanho quando suspensa em meio de contraste não iônico ou quando suspensa em uma solução alternativa de contraste não iônico e álcool absoluto, e concluíram que a partícula de PVA-flocular aumentou significativamente de tamanho nas duas diferentes soluções, uma vez que o PVAflocular é uma espuma de polivinil que se comporta como esponja. Choe et al. realizaram um estudo para avaliar os efeitos embólicos de acordo com a velocidade de infusão e com a concentração de PVA-flocular em suspensão, e concluíram que infusões mais lentas de suspensões mais diluídas são capazes de promover oclusão arterial mais distal, uma vez que as partículas de PVA-flocular estão mais dispersas na solução e as chances de agregação são menores ${ }^{15}$.

As partículas de PVA inicialmente usadas nas formas de espuma ou esponja também apresentavam outros inconvenientes como difícil preparo e apresentações com grande variação no tamanho das partículas. As partículas em apresentações iniciais de apenas 250-590 ou 590-1000mm de diâmetro eram ainda preparadas em formalina e previamente à embolização deveriam ser lavadas com solução estéril para eliminar qualquer resíduo. Em seguida, passaram a ser cortadas em tamanhos predeterminados e manobras foram criadas com intuito de se evitar agregação das partículas, como a utilização de um misturador para mantê-las uniformemente em suspensão $0^{16}$.

Acreditando-se que a forma irregular das partículas de PVA-flocular seja a responsável pela agregação e aglutinação das mesmas, uma variedade de agentes embólicos de forma esférica foi desenvolvida. Os agentes têm sido desenvolvidos a partir de variados materiais orgânicos ou inorgânicos, incluindo colágeno, dextran e polímero de trisacril impregnado com gelatina ${ }^{10,17,18}$.

Procurando produzir um agente embólico inerte, com maior resistência à degradação e sem os efeitos indesejáveis decorrentes da superfície irregular das partículas de PVAflocular vários ensaios foram realizados na COPPE/UFRJ com a utilização de mecanismos de iniciação alternativos, como a aplicação de luz ionizante ultravioleta, propondo novas rotas viáveis de síntese de PVA para utilização na embolização vascular $^{19}$. Também foram considerados os procedimentos clássicos de polimerização de acetato de vinila (precursor do PVA) a quente, seguido de hidrólise via soluções básicas e etapas de purificação, além da polimerização do acetato de vinila a frio em câmara de ultravioleta, com controle do teor de ramificações do PVAc. Desta forma foi possível desenvolver uma nova partícula com tecnologia exclusivamente nacional, composta de uma fina superfície de PVAe um núcleo de PVAc, com morfologia esférica, não hidrossolúvel e consequentemente mais resistente à biodegradação, além de viabilizar apresentações nos variados tamanhos desejados.

Durante a avaliação microscópica dos dois tipos de partículas na COPPE/UFRJ, as partículas de PVA+PVAc foram observadas serem uniformemente esféricas e com superfícies lisas em todas as amostras avaliadas e nos diferentes diâmetros produzidos. Nenhum pequeno fragmento foi notado. Em comparação com as partículas de PVA-flocular, estas geralmente apresentam forma irregular floculada, com projeções anguladas irregulares e pontiagudas e em suspensão alguns pequenos fragmentos foram observados.

As partículas de PVA+PVAc-esférico apresentaram passagem pelo cateter mais fácil que as partículas de PVAflocular. Aglomeração e oclusão do cateter, que ocorreu em algumas utilizações com o PVA-flocular, não foi observado com as partículas de PVA+PVAc-esférico. Estas diferenças podem ser atribuídas a superfície lisa das partículas de PVA+PVAc-esférico.

Derdeyn et al. ${ }^{4}$ em estudo experimental comparativo entre as partículas de PVA-flocular e as partículas microesféricas de acrílico revestidas com colágeno de mesmo tamanho (Embosphere ${ }^{\circledR}$, Guerbet Biomedical, Louvres, France) observaram diferença significativa no grau de penetração das partículas. Enquanto as partículas de PVA-flocular freqüentemente se agregavam na artéria faríngea ascendente de porcos, as partículas microesféricas atingiam e ultrapassavam a microcirculação. Estes autores atribuíram esta diferença às características das partículas microesféricas que apresentam superfície lisa e hidrofílica, deformável e não se agregam. Referem ainda que estas características sejam responsáveis pela facilidade na injeção das partículas microesféricas e pela não acumulação central e oclusão do cateter. O grau de penetração das partículas de PVA-flocular dentro do sistema vascular pode ser afetado por muitos fatores $^{8}$. O tamanho anunciado de uma partícula de PVA-flocular é primariamente uma função do seu eixo intermediário, o qual é responsável pela habilidade da partícula passar através dos orifícios quadrados da peneira responsável pela separação da partícula de PVA-flocular por tamanhos. E quando o diâmetro do vaso é pequeno para o diâmetro intermediário da partícula ela se acunha no vaso ${ }^{8}$.

Entretanto, Quisling et $a l^{20}$ mostraram que as partículas de PVA-flocular tendem a aderir nas paredes dos vasos de maior diâmetro antes que venham a se acunhar em vaso distal. Além disso, as partículas de PVA-comercial tendem a se amontoar e agregar dentro do cateter. Acumulações similares 
podem ocorrer dentro do vaso no local de aderência das partículas. Por estes motivos, a oclusão do vaso com as partículas de PVA-flocular pode ocorrer freqüentemente em local mais proximal que o esperado para uma dada partícula e certo diâmetro da luz do vaso.

As partículas esféricas de PVA+PVAc desenvolvidas na UFRJ foram observadas experimentalmente e mostraram subjetivamente penetrar mais distalmente no vaso que as partículas de PVA-flocular e puderam ser injetadas com menor dificuldade. Beaujeux et $a l^{21}$ notaram que as partículas microesféricas deveriam ser utilizadas em tamanhos maiores que o previsto para se ocluir completamente um vaso durante uma embolização. E atribuíram esta necessidade ao fato que a escolha inicial por um determinado tamanho de partícula era baseado na experiência da equipe médica com as partículas de PVA-flocular.

O maior grau de penetração observado com as partículas de PVA+PVAc-esférico pode ser vantajoso ao realizar uma embolização transarterial mais efetiva de muitas lesões. Entretanto, em lesões com evidência de comunicação artériovenosa deverão ser necessários maiores estudos, uma vez que foi relatado em estudo com as partículas microesféricas, que estas podem atravessar a lesão e promover embolização pulmonar. $\mathrm{O}$ maior grau de penetração observado com as partículas de PVA+PVAc-esférico pode também ser atribuído à sua capacidade de compressibilidade, adaptando-se à conformação do vaso, podendo assim atingir vasos mais distais.

Em ambos os grupos, os agentes (flocular e esférico) foram efetivos promovendo grande isquemia dos rins, com subseqüentes fases inflamatórias aguda e crônica, evoluindo posteriormente para fibrose do órgão. Os tempos para morte e retirada dos órgãos foram baseados nas diferentes fases de reação inflamatória, já estabelecidas em estudos microscópicos prévios de coração com infarto do miocárdio. O propósito deste estudo foi estudar a reação inflamatória que ocorre no rim com infarto renal promovido por oclusão completa da sua vascularização, com os dois tipos diferentes de agentes embólicos estudados.

As partículas de PVA-flocular promoveram resposta inflamatória moderada enquanto que as partículas de PVA+PVAc-esférico promoveram reação intensa, o que pode ser devido ao maior grau de penetração da partícula, além de corresponder às reações ao trombo e à oclusão do vaso propriamente dita.
As partículas de PVA+PVAc-esférico assim como as de PVA-flocular quando não promovem oclusão, não promovem qualquer reação inflamatória, ou seja, são inertes. Sendo a resposta inflamatória somente observada quando os vasos são completamente ocluídos ${ }^{4,22}$.

A oclusão do vaso promovida pelas partículas de PVA-flocular, que antigamente era considerada permanente, sabe-se hoje, que não ocorre. As partículas de PVA são biodegradáveis e a recanalização do vaso ocorre ${ }^{6,10}$. Os resultados histopatológicos obtidos neste estudo, mostram que as partículas de PVA-flocular sofreram uma grande retração nas peças de 30 dias.

As partículas de PVA-flocular, como apresentam forma muito irregular, permitem que se formem entre elas espaços que vão ser preenchidos por sangue. $O$ sangue após a oclusão do vaso, coagula, se torna um trombo, que durante a fase de reação inflamatória é reabsorvido. Associado a isto, a degradação das partículas de PVA-flocular, que também ocorre, vai criar mais espaços e favorecer ainda mais a recanalização do vaso.

No exame das lâminas nas peças observou-se que as partículas de PVA+PVAc-esférico apresentaram melhor adaptação entre elas e o vaso, promovendo assim a menor formação de espaços para serem preenchidos por sangue, sendo também menor a quantidade de sangue nos espaços, não suficiente para favorecer a recanalização do vaso.

$\mathrm{O}$ estudo anatomopatológico mostra ainda que a reação inflamatória promovida com a oclusão do vaso pelas partículas de PVA+PVAc-esférico ocorreram em vasos mais distais e foram mais intensas do que com as partículas de PVA-flocular, promovendo crescimento celular para dentro da luz do vaso, com consequiente fibrose e dificultando, assim, qualquer chance de recanalização.

Nossos resultados sugerem que a partícula de PVA+PVAc-esférico é um agente embólico efetivo, fácil de passar pelo cateter e que não se aglutina nos vasos, alcançando segmentos arteriais mais distais que as partículas de PVA comercialmente disponíveis. De acordo com os resultados experimentais obtidos, a partícula de PVA+PVAc-esférico apresenta propriedades físicas e biológicas desejáveis para um agente de embolização, sendo uma atraente alternativa para as embolizações intra-arteriais transcateter.

\begin{abstract}
Background: To evaluate the characteristics and the effects of an embolic agent, available commercially, consisting of irregular - Polyvinyl Alcohol (PVA), and to compare with a spherical agent, of brazilian technology, consisting of Polyvinyl Alcohol and Polyvinyl Acetate (PVA + PVAc). Methods: Renal arterial embolization was performed in females of New Zealand White rabbits. Irregular - PVA was used in 24 animals. Spherical - PVA+PVAc was used in 24 animals. Six animals were used as control. All animals were maintained in captivity until the euthanasia, after 48 hours, 5 days, 10 days and 30 days. Results: Both agents resulted in vessel occlusion and organ infarction. The initial macroscopic study of the arteries embolized with irregular-PVA, the occluding plug consisted of thrombus and PVA. In vessels embolized with spherical-PVA+PVAc, the occluding plug consisted mostly of the embolic agent. After 30 days, there is absorption of the thrombus and retraction of the agents of PVA-irregular, creating spaces. With spherical-PVA+PVAc, it can be observed the agents surrounded by intense fibrosis. Conclusions: Both particles were effective to cause tissue ischemia. The inflammatory reaction was more intense with spherical-PVA $+P V A c$, besides presenting larger degree of penetration in the vascular system.
\end{abstract}

Key-words: Animal experimentation; Embolization, therapeutic; Polyvinyl alcohol; Radiology, interventional. 


\section{REFERÊNCIAS}

1. Siskin GP, Dowling K, Virmani R, et al. Pathologic evaluation of a spherical polyvinyl alcohol embolic agent in a porcine renal model. J Vasc Interv Radiol. 2003;14(1):89-98.

2. Kerber CW, Bank WO, Horton JA. Polyvinyl alcohol foam: prepackaged emboli for therapeutic embolization. AJR Am J Roentgenol. 1978;130(6):1193-4.

3. Latchaw RE, Gold LH. Polyvinyl foam embolization of vascular and neoplastic lesions of the head, neck and spine. Radiology. 1979;131(3):669-79.

4. Derdeyn CP, Graves VB, Salamat MS, et al. Collagen-coated acrylic microspheres for embolotherapy: in vivo and in vitro characteristics. AJNR Am J Neuroradiol. 1997;18(4):647-53.

5. Tadavarthy SM, Moller JH, Amplatz K. Polyvinyl alcohol (Ivalon) - a new embolic material. Am J Roentgenol Radium Ther Nucl Med. 1975;125(3):609-16.

6. Castaneda-Zuniga WR, Sanchez R, Amplatz K. Experimental observations on short and long-term effects of arterial occlusion with Ivalon. Radiology. 1978;126(3):783-5.

7. Jack CR, Forbes G, Dewanjee MK, et al. Polyvinyl alcohol sponge for embolotherapy: particle size and morphology. AJNR Am J Neuroradiol. 1985;6(4):595-7.

8. Derdeyn CP, Moran CJ, Cross DT, et al. Polyvinyl alcohol particle size and suspension characteristics. AJNR Am J Neuroradiol. 1995;16(6):1335-43.

9. Pelage JP, Laurent A, Wassef M, et al. Uterine artery embolization in sheep: comparison of acute effects with polyvinyl alcohol particles and calibrated microspheres. Radiology. 2002;224(2):436-45.

10. Dion JE, Rankin RN, Viñuela F, et al. Dextran microsphere embolization: experimental and clinical experience with radiologic-pathologic correlation. Work in progress. Radiology. 1986;160(3):717-21.

11. Wright KC, Greff RJ, Price RE. Experimental evaluation of cellulose acetate NF and ethylene-vinyl alcohol copolymer for selective arterial embolization. J Vasc Interv Radiol. 1999;10(9):1207-18.

12. Spies JB, Benenati JF, Worthington-Kirsch RL, et al. Initial experience with use of tris-acryl gelatin microspheres for uterine artery embolization for leiomyomata. J Vasc Interv Radiol. 2001;12(9):1059-63.
13. Binkert CA, Andrews RT, Pavcnik D, et al. Lack of suitability of the rabbit model for particulate uterine artery embolization. J Vasc Interv Radiol. 2002;13(6):609-12.

14. Kubo M, Kuwayama N, Hirashima Y, et al. Hydroxyapatite ceramics as a particulate embolic material: report of the physical properties of the hydroxyapatite particles and the animal study. AJNR Am J Neuroradiol. 2003;24(8):1540-4.

15. Choe DH, Han MH, Kang GH, et al. An experimental study of embolic effect according to infusion rate and concentration of suspension in transarterial particulate embolization. Invest Radiol. 1997;32(5):260-7.

16. Berenstein A, Graeb DA. Convenient preparation of ready-touse particles in polyvinyl alcohol foam suspension for embolization. Radiology. 1982;145(3):846.

17. Turjman F, Massoud TF, Vinters HV, et al. Collagen microbeads: experimental evaluation of an embolic agent in the rete mirabile of the swine. AJNR Am J Neuroradiol. 1995;16(5):1031-6.

18. Laurent A, Beaujeux R, Wassef $M$, et al. Trisacryl gelatin microspheres for therapeutic embolization, I: development and in vitro evaluation. AJNR Am J Neuroradiol. 1996;17(3):53340.

19. Yamamoto T, Seki S, Fukae R, et al. High molecular weight poly(vinyl alcohol) through photo-emulsion polymerizations of vinyl acetate. Polymer J. 1990;22:567-71.

20. Quisling RG, Mickle JP, Ballinger WB, et al. Histopathologic analysis of intraarterial polyvinyl alcohol microemboli in rat cerebral cortex. AJNR Am J Neuroradiol. 1984;5(1):101-4.

21. Beaujeux R, Laurent A, Wassef M, et al. Trisacryl gelatin microspheres for therapeutic embolization, II: preliminary clinical evaluation in tumors and arteriovenous malformations. AJNR Am J Neuroradiol. 1996;17(3):541-8.

22. White RI, Strandberg JV, Gross GS, et al. Therapeutic embolization with long-term occluding agents and their effects on embolized tissues. Radiology. 1977;125(3):677-87.

Endereço para Correspondência:

Warley Dias Siqueira Mendes

Rua: Barão de Itapagipe, 445 / 306

Tijuca

20261-000 - Rio de Janeiro - RJ 